ARTICLE

https://doi.org/10.1038/s41467-019-12846-7

\title{
Identification of predictors of drug sensitivity using patient-derived models of esophageal squamous cell carcinoma
}

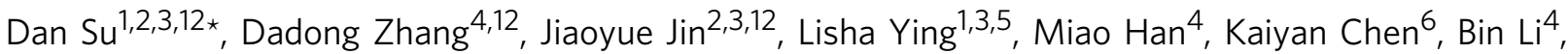 \\ Junzhou Wu (1) 1,5, Zhenghua Xie ${ }^{4}$, Fanrong Zhang7 , Yihui Lin ${ }^{4}$, Guoping Cheng ${ }^{2}$, Jing-Yu Li ${ }^{4}$, Minran Huang 1,3,5, \\ Jinchao Wang ${ }^{4}$, Kailai Wang (10 ${ }^{1}$, Jianjun Zhang ${ }^{8,9}$, Fugen $\mathrm{Li}^{4}$, Lei Xiong ${ }^{4}$, Andrew Futreal ${ }^{8,10}$ \& Weimin Mao ${ }^{1,3,11 \star}$
}

Previous studies from the Cancer Cell Line Encyclopedia (CCLE) project have adopted commercial pan-cancer cell line models to identify drug sensitivity biomarkers. However, drug sensitivity biomarkers in esophageal squamous cell carcinoma (ESCC) have not been widely explored. Here, eight patient-derived cell lines (PDCs) are successfully established from 123 patients with ESCC. The mutation profiling of PDCs can partially recapture the tumor tissue actionable mutations from 161 patients with ESCC. Based on these mutations and relative pathways in eight PDCs, 46 targeted drugs are selected for screening. Interestingly, some drug and biomarker relationships are established that were not discovered in the CCLE project. For example, CDKN2A or CDKN2B loss is significantly associated with the sensitivity of CDK4/6 inhibitors. Furthermore, both PDC xenografts and patient-derived xenografts confirm CDKN2A/2B loss as a biomarker predictive of CDK4/6 inhibitor sensitivity. Collectively, patient-derived models could predict targeted drug sensitivity associated with actionable mutations in ESCC.

\footnotetext{
${ }^{1}$ Institute of Cancer and Basic Medicine (ICBM), Chinese Academy of Sciences, Hangzhou, Zhejiang, China. ${ }^{2}$ Department of Pathology, Cancer Hospital of the University of Chinese Academy of Sciences, Hangzhou, Zhejiang, China. ${ }^{3}$ Zhejiang Cancer Hospital, Hangzhou, Zhejiang, China. ${ }^{4}$ Research and Development Institute of Precision Medicine, 3D Medicines Inc., Shanghai, China. ${ }^{5}$ Cancer Hospital of the University of Chinese Academy of Sciences, Hangzhou, Zhejiang, China. ${ }^{6}$ Department of Chemotherapy, Cancer Hospital of the University of Chinese Academy of Sciences, Hangzhou, Zhejiang, China. ${ }^{7}$ Department of Breast Surgery, Cancer Hospital of the University of Chinese Academy of Sciences, Hangzhou, Zhejiang, China. ${ }^{8}$ Department of Genomic Medicine, University of Texas MD Anderson Cancer Center, Houston, Texas, USA. ${ }^{9}$ Department of Thoracic/Head and Neck Medical Oncology, University of Texas MD Anderson Cancer Center, Houston, Texas, USA. ${ }^{10}$ Honorary Faculty, Wellcome Trust Sanger Institute, Hinxton, UK. ${ }^{11}$ Department of Thoracic Surgery, Cancer Hospital of the University of Chinese Academy of Sciences, Hangzhou, Zhejiang, China. ${ }^{12}$ These authors contributed equally: Dan Su, Dadong Zhang, Jiaoyue Jin. *email: sudan@zjcc.org.cn; Weimin.mao@zjcc.org.cn
} 
S ystemic studies of chemical compounds and genomic alternation have been conducted thoroughly by screening a large set of compounds against hundreds of commercial cell lines ${ }^{1-3}$. Many known agents associated with driven gene mutations were re-confirmed such as gefitinib and the EGFR (epidermal growth factor receptor) mutation, and some relationships, which have not been reported before, were also discovered ${ }^{1-3}$. In the meantime, these relationships could be limited by multiple cell lines across pan caner types due to heterogeneity and the loss of originality of commercial cell lines. For example, Garnett et al. ${ }^{2}$ adopted a pan-cancer cell model to perform a systematic identification of genomic markers of drug sensitivity, which might be limited in the clinical study of a single type of cancer.

Commercial cell lines are limited and might lose their original tumor characteristics due to repeated passaging, which results in genetic variation and divergence from the original tumor ${ }^{4}$. Therefore, cell biology assays and xenograft mouse models based on commercial cell lines might not be informative enough ${ }^{5}$. Patient-derived cell lines (PDCs) with low passage could be good alternatives to commercially available cell lines because they are directly derived from fresh tumor tissues ${ }^{6,7}$, inheriting the complexity and genetic diversity of the original tumor ${ }^{8,9}$. As a valuable experimental material, the success rate of PDC establishment directly isolated from tumor tissue samples is low ${ }^{10,11}$. Our previous study demonstrated that PDC-based models have been applied to elucidate the sensitivity of cells to various therapeutic agents $^{12}$. Additionally, patient-derived xenografts (PDXs) are created when patient-derived cancerous tissue without prior digestion or in vitro culture are implanted directly into an immunodeficient mouse and could be used as extensively annotated models for pre-clinical analysis of therapeutics ${ }^{13-15}$. Hence, this study established an approach using patient-derived cells and models to explore the biomarkers of drug sensitivity and validate the results in esophageal squamous cell carcinoma (ESCC).

ESCC is the third most common cancer type in China ${ }^{16}$. Despite recent improvements in multimodal therapy, the 5-year overall survival (OS) rate of ESCC patients is only $25-40 \% 17$. There are currently no effective targeted drugs approved for ESCC. Recent genomic studies on ESCC have revealed frequently mutated cancer genes ${ }^{18-24}$, as well as recurrent somatic copy number variations (CNVs) at $11 \mathrm{q} 13.2-\mathrm{q} 13.4$ and $9 \mathrm{p} 21.3^{23}$. Although uncovering of the genomic landscape and functional study of these mutant cancer genes have deepened our understanding of the mechanism of ESCC occurrence and development, further exploration and validation of these genes as potential therapeutic biomarkers of drug sensitivity for ESCC are largely lacking. In particular, an effective method of biomarker exploration and validation is absent.

A number of drugs for screening were limited in the actionable mutations and related pathways ${ }^{25-28}$. In this study, only targeted deep sequencing focused on tumor-related genes was an effective approach to identifying genomic variants associated with cancer tumorigenesis in a large cohort of ESCC and their PDCs. One aim is to understand the landscape of tumor-related genomic alternations in ESCC and another is to discover the actionable mutations for drug screening in PDCs to establish the relationship between drug and mutation. The selected biomarker and corresponding drug was further validated in vitro and in vivo. This study demonstrated that deep sequencing combined with patient-derived models can identify potential biomarkers of targeted drug sensitivity in ESCC.

\section{Results}

Cancer gene mutations in ESCC. To investigate the somatic cancer gene mutation landscape of ESCCs, we performed next-

\section{Table 1 Clinicopathological features of 161 patients for profiling cancer gene mutations}

\begin{tabular}{|c|c|c|}
\hline Factors & No. of patients & $\%$ \\
\hline \multicolumn{3}{|l|}{ Gender } \\
\hline Male & 140 & 87.0 \\
\hline Female & 21 & 13.0 \\
\hline \multicolumn{3}{|l|}{ Age (years) } \\
\hline$\leq 65$ & 128 & 79.5 \\
\hline$>65$ & 33 & 20.5 \\
\hline Mean, median (range) & $60.2,61.0(43-79)$ & \\
\hline \multicolumn{3}{|c|}{ Body mass index $\left(\mathrm{kg} / \mathrm{m}^{2}\right)$} \\
\hline$<18.5$ & 30 & 18.6 \\
\hline $18.5-25$ & 119 & 73.9 \\
\hline$>25$ & 11 & 6.8 \\
\hline Missing & 1 & 0.6 \\
\hline Mean, median (range) & $21.0,20.8(15.4-29.3)$ & \\
\hline \multicolumn{3}{|l|}{ Smoking history } \\
\hline No & 36 & 22.4 \\
\hline Yes & 124 & 77.0 \\
\hline Missing & 1 & 0.6 \\
\hline \multicolumn{3}{|l|}{ Alcohol intake } \\
\hline No & 43 & 26.7 \\
\hline Yes & 117 & 72.7 \\
\hline Missing & 1 & 0.6 \\
\hline \multicolumn{3}{|l|}{ Family history } \\
\hline No & 112 & 69.6 \\
\hline Yes & 48 & 29.8 \\
\hline Missing & 1 & 0.6 \\
\hline \multicolumn{3}{|l|}{ Tumor location } \\
\hline Upper & 13 & 8.1 \\
\hline Middle & 96 & 59.6 \\
\hline Lower & 52 & 32.3 \\
\hline \multicolumn{3}{|l|}{ Grade } \\
\hline Well & 2 & 1.2 \\
\hline Moderate & 118 & 73.3 \\
\hline Poor & 37 & 23.0 \\
\hline Missing & 4 & 2.5 \\
\hline \multicolumn{3}{|l|}{ Clinical stage } \\
\hline I & 1 & 0.6 \\
\hline II & 4 & 2.5 \\
\hline Illa & 91 & 56.5 \\
\hline IIIb & 47 & 29.2 \\
\hline IIllc & 18 & 11.2 \\
\hline
\end{tabular}

generation sequencing (NGS) of 161 tumor samples paired with a matching peripheral blood sample as a normal control, on a panel targeting 365 tumor-associated genes (Table 1, Supplementary Data 1 and 2). The mean sequencing depth of the tumors and of the matched blood DNA samples were $394 \times$ and $431 \times$, respectively (Supplementary Data 3). A total of 2121 unique somatic mutations (Supplementary Data 4) was discovered with 57\% missense mutations, $30 \%$ synonymous mutations, $7 \%$ stop-gain mutations, and 3\% splice sites (Supplementary Fig. 1a), which are compatible with previous studies ${ }^{18-20}$. The most recurrent base substitution is the $\mathrm{C}>\mathrm{T}$ transition (Supplementary Fig. 1b).

The most frequently mutated genes in the cohort of 161 samples (Fig. 1a) were also frequently observed in previous studies of ESCC ${ }^{18-21,23}$. These included TP53, KMT2D (MLL2), KMT2C (MLL3), NOTCH1, LRP1B, EP300, PIK3CA, FAT1, CREBBP, ADAM29, RB1, NOTCH2, and others (Fig. 1a). Out of 161 ESCCs, 153 (95\%) samples have at least one somatic CNV. Within these CNVs, eight CNVs have recurrence rates $\geq 30 \%$ (Fig. 1b and Supplementary Data 5). Among all genes with copy number alterations, CCND1 (42\%), MCL1 (38\%), FGF4 (35\%), FGF3 (35\%), SOX2 (34\%), FGF19 (34\%), and CDKN1B (30\%) were frequently amplified, while MST1R (30\%), CDKN2A (26\%), and $C D K N 2 B(13 \%)$ were recurrently deleted (Fig. $1 \mathrm{~b}$ ). 

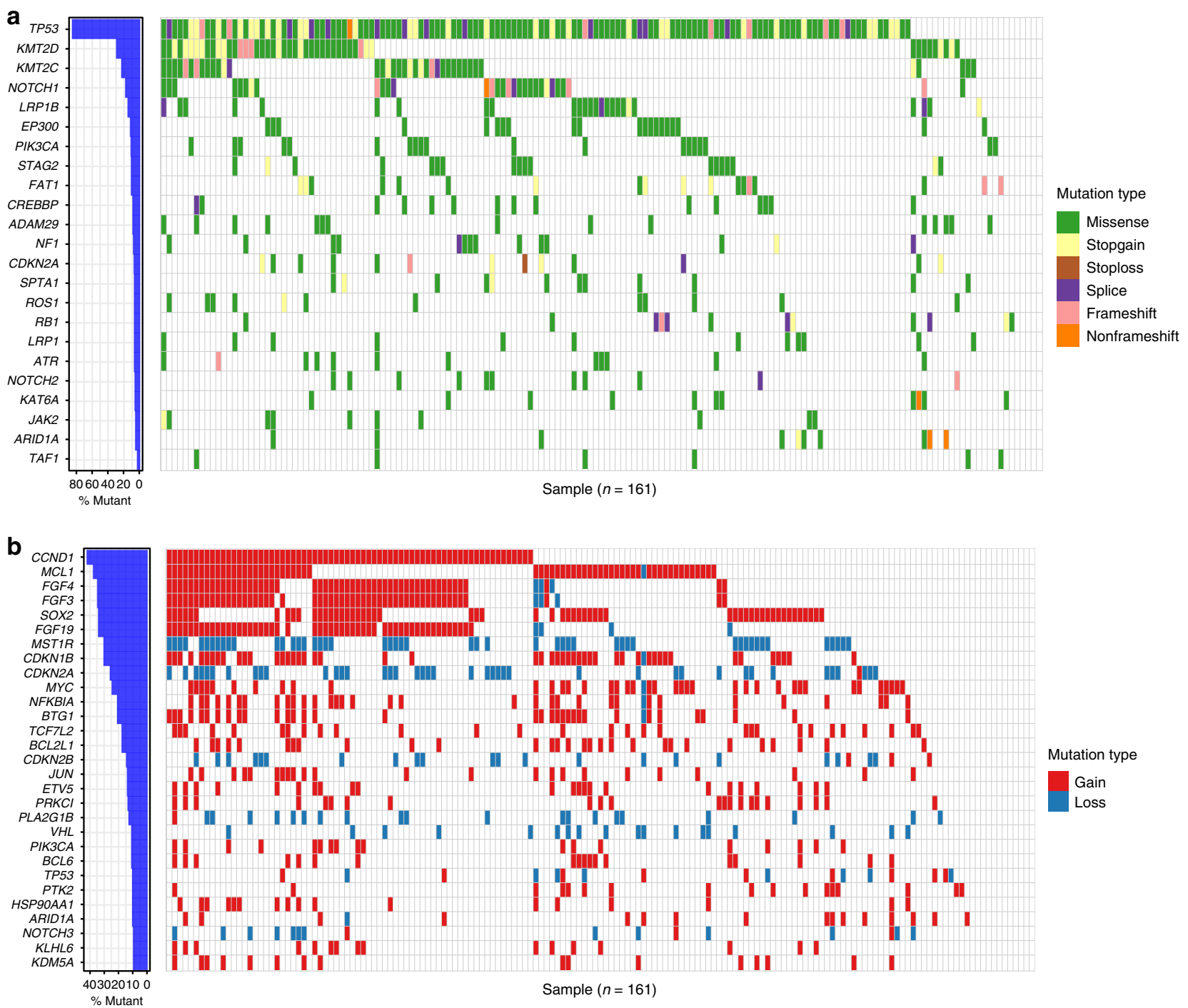

Fig. 1 Top recurrent genes harbored somatic variants and somatic CNVs. a Left panel, bar plot shows the proportion of 161 ESCC samples with somatic mutations in the specific genes. Right panel, occurrence of the top 23 ranked somatically mutated genes identified by the cancer panel. Mutation subtypes (Missense, Stopgain, Stoploss, Splice, Frameshift, and Non-frameshift) are denoted by color. b Heatmap of the top recurrent genes associated with the top recurrent somatic CNVs in the 161 ESCC samples. The genes with recurrence of more than $10 \%$ are shown here. Mutation subtypes (Gain and Loss) are denoted by color

To investigate the associations of mutations with clinical outcomes, a survival analysis based on the genetic profile of the 161 ESCC patients was carried out. We found that PIK3CA amplification and JUN neutral were significantly associated with poorer disease-free survival (DFS, $p=0.036$ and 0.043 , log-rank test) and OS (OS, $p=0.023$ and 0.018, log-rank test) (Supplementary Fig. 2a, b). ESCC patients with $H 3 F 3 A$ amplification had a significantly shortened DFS $(p=0.003)$ and a trend of shortened OS ( $p=0.160$, log-rank test) in comparison to those harboring H3F3A neutral (Supplementary Fig. 2c). ESCC patients with NOTCH2 mutations had a significantly shortened OS in comparison to those without NOTCH 2 mutations ( $p=0.034$, logrank test, Supplementary Fig. 2d). In addition, we found that ACVR2A amplification was associated with poorer DFS ( $p=$ 0.038 , log-rank test, Supplementary Fig. 2e), while DAXX amplification was associated with poorer OS $(p=0.050$, logrank test, Supplementary Fig. 2f).

Study of the potential biomarkers of drug sensitivity. To further explore the potential biomarkers of drug sensitivity in ESCC, establishment and molecular characterization of PDCs were integrated with deep sequencing and drug sensitivity evaluation into an approach (Fig. 2a). We succeeded in deriving eight ESCC PDCs (ZEC043, ZEC056, ZEC061, ZEC118, ZEC127, ZEC145, ZEC157, and ZEC166) from the 123 ESCC patient tissues available, with a $6.5 \%$ success rate of establishing ESCC PDCs (Supplementary Data 6). Cell morphology, identification, and imaging results of karyotyping are distinct across PDCs (Supplementary Fig. 3a, b and Supplementary Data 7). Karyotype examination demonstrated that the number of chromosomes ranged from 34 to 85. Furthermore, comparison analyses of short tandem repeats (STRs) of these eight ESCC PDCs (Supplementary Data 8) with those from American Type Culture Collection, Deutsche Sammlung von Mikroorganismen und Zellkulturen, and other cell banks suggested that all eight ESCC PDCs are unique lines, devoid of cross-contamination with other known cancer cell lines. To confirm the origin of these PDCs, single-nucleotide polymorphisms (SNP) analysis demonstrated that SNPs in the PDCs were clustered with those in the corresponding tumor tissues (Supplementary Fig. 3c), confirming that the PDCs are derived 


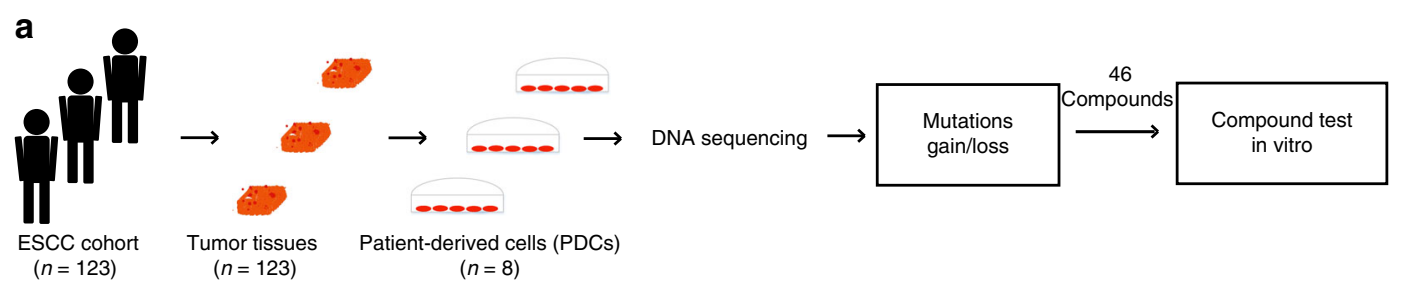

b

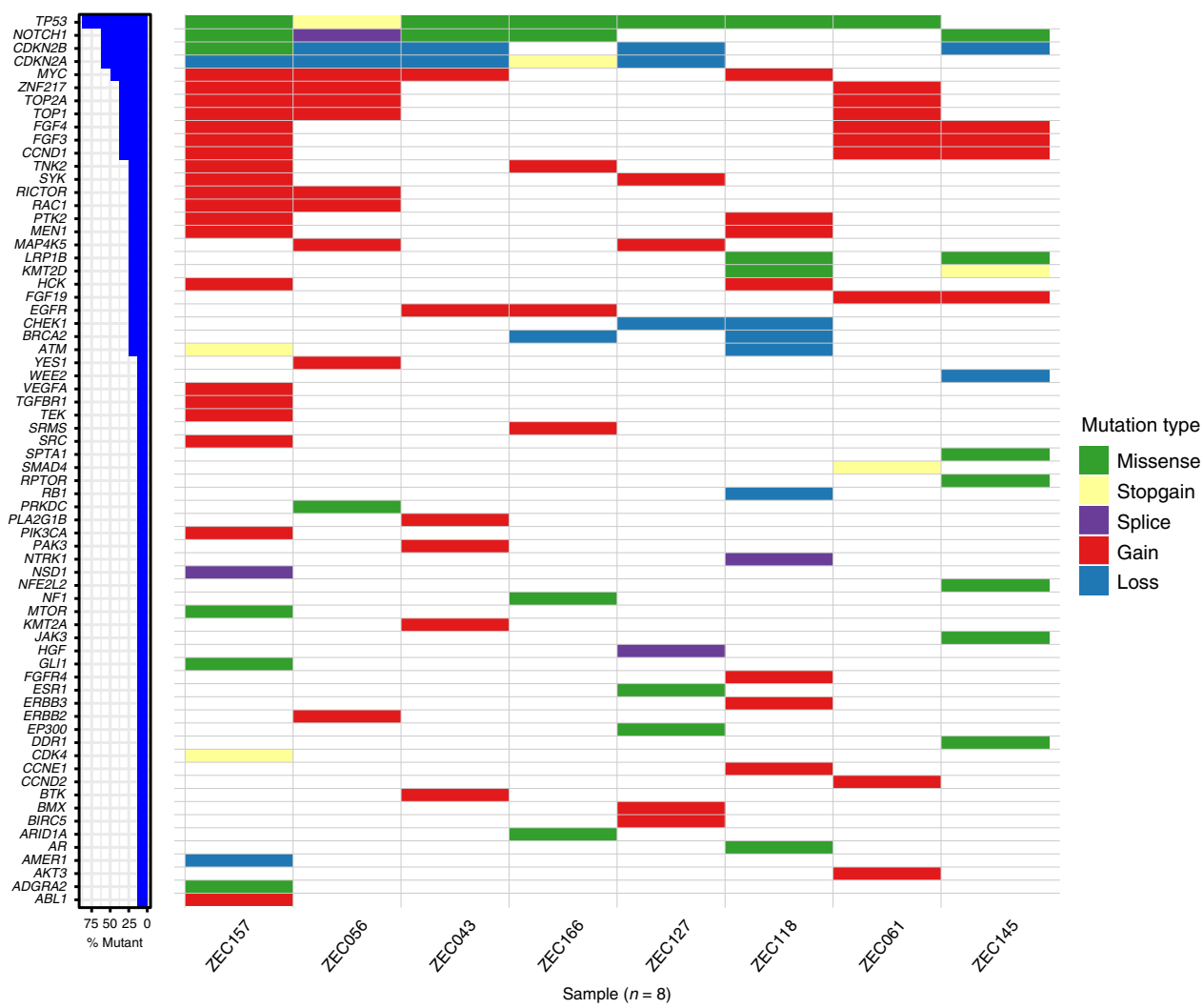

C

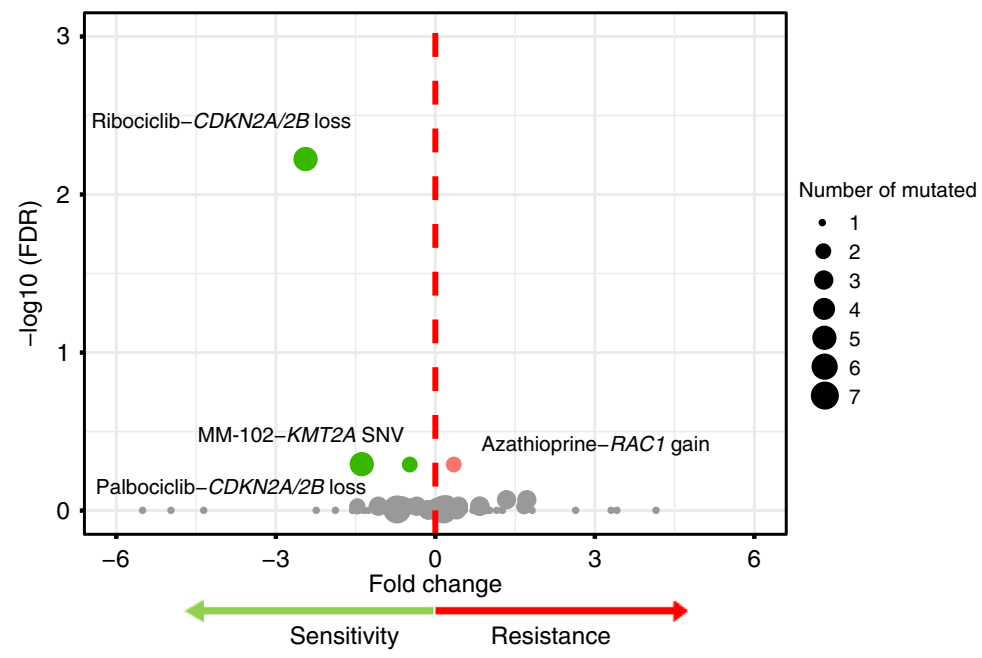

Fig. 2 Exploration of the potential biomarkers of drug sensitivity in ESCC. a A schematic diagram exploring potential biomarkers of drug sensitivity in esophageal squamous cell carcinoma. Eight ESCC patient-derived cells (PDCs) were established from an independent cohort of 123 ESCC patients. DNA sequencing was used to detect the mutations and gain/loss in ESCC PDCs. We used the ESCC PDCs for integrated targeted deep sequencing and drug sensitivity evaluation systems to explore potential biomarkers of drug sensitivity. b The mutational landscape was studied in eight ESCC PDCs. $\mathbf{c}$ Each circle represents a single drug-gene interaction, and the size is proportional to the number of mutant cell lines screened (range 1-7). An unpaired $t$ test was performed for each drug-gene mutation associations. The top four drug-gene mutation associations sorted by FDR were colored by green (sensitive) and red (resistant) 
from their corresponding tumor tissues without cross-sample contamination.

The somatic mutational landscape of these eight PDCs was profiled using targeted deep sequencing (Fig. 2b, Supplementary Data 9 and 10). These PDCs recaptured some mutational characteristics of the ESCC population. For example, the eight PDCs harbored the single-nucleotide variant (SNV)/indel overlapping genes found in the 161 ESCC patients (recurrence $\geq 5 \%$ ), including $A R, A R I D 1 A, C D K N 2 A, E P 300, K M T 2 D, L R P 1 B, N F 1$, NOTCH1, SPTA1, and TP53 (Supplementary Fig. 4a). In addition, the CNV overlapping genes between the eight PDCs and the 161 ESCC patients (recurrence $\geq 10 \%$ ) contained $M Y C, F G F 19, P T K 2$, CDKN2A, CDKN2B, CCND1, PLA2G1B, HSP90AA1, PIK3CA, FGF3, and FGF4 (Supplementary Fig. 4b). These results showed that ESCC PDCs could partially recapture the mutational characteristics of tumor tissues from 161 ESCC patients.

Based on the mutational landscape of the eight PDCs, a list of 46 compounds targeting mutated genes or their pathways was identified for a drug-sensitivity evaluation (Supplementary Data 11). The effect of $96 \mathrm{~h}$ of drug treatment on cell viability was used to derive the half-maximal inhibitory concentration (IC50) of drug sensitivity. In total, the IC50s of 46 compounds on ESCC PDCs ranged from 0.002 to $110.273 \mu \mathrm{M}$ (Supplementary Data 12). To study the associations between the mutated genes and drug sensitivity across the ESCC PDCs, we used an analysis incorporating the ratio and significance of IC50s between mutated PDCs and non-mutated ones. This analysis revealed that there was an obvious gap of false discovery rate (FDR) level between top four drug-mutation associations sorted by FDR and other associations. The top four drug-mutation associations that have been preliminarily established were considered as the candidates (Fig. 2c and Supplementary Data 13). Interestingly, the two drug and biomarker relationships in four top candidates are associations involving $C D K N 2 A$ or $C D K N 2 B$ loss and CDK4/ 6 inhibitors (ribociclib and palbociclib). Moreover, there were five PDCs harboring CDKN2A or CDKN2B loss, three PDCs carrying no CNVs of $C D K N 2 A$ and $C D K N 2 B$. Therefore, we focused on $C D K N 2 A$ or $C D K N 2 B$ loss as a potential biomarker of CDK4/6 inhibitor sensitivity.

Validation of the biomarkers of drug sensitivity in vitro. For the CNVs of CDKN2A and CDKN2B in tumor tissue, we found that $C D K N 2 A$ or $C D K N 2 B$ loss was detected in $27 \%(44 / 161)$ of the ESCC samples (Fig. 1b). CDKN2A loss occurred in 41 ESCC formalin-fixed and paraffin-embedded (FFPE) samples (25\%, 41/ $161)$, while $C D K N 2 B$ loss was detected in 21 samples $(13 \%, 21 /$ $161)$, with loss of both being found in 18 samples $(11 \%, 18 / 161)$ (Supplementary Fig. 5a). It has been suggested that CDKN2A and $C D K N 2 B$ tend to be lost together.

To further validate potential CDK4/6 inhibitor sensitivity conferred by CNVs of CDKN2A and CDKN2B, two CDK4/6 inhibitors, palbociclib (PD-0332991) and ribociclib (LEE011), approved for clinical breast cancer use, were used to perform an analysis on the sensitivity of eight PDCs. We found that five out of eight PDCs (ZEC043, ZEC056, ZEC145, ZEC127, and ZEC157) harbored CDKN2A loss or CDKN2B loss (Fig. 3a). Due to intratumoral heterogeneity of tumor tissue and clonal enrichment of PDCs, some PDCs were not completely concordant with corresponding tumor tissue in detecting the CNVs of CDKN2A and CDKN2B (Fig. 3a). The average IC50s of palbociclib and ribociclib ranged from 0.361 to $28.669 \mu \mathrm{M}$ and from 2.314 to $61.806 \mu \mathrm{M}$, respectively, in the eight ESCC PDCs (Supplementary Data 14). Interestingly, the IC50s of palbociclib ( $p=0.0395$, Student's $t$ test) in the CDKN2A or the CDKN2B loss group were significantly lower than in the wild-type group, while the IC50s of ribociclib ( $p=0.0043$, Student's $t$ test) in the $C D K N 2 A$ or $C D K N 2 B$ loss group were also significantly lower than in the wild-type group (Fig. 3b). In addition, there was no clinical characteristic of PDC corresponding patients associated with the sensitivities of palbociclib and ribociclib (Supplementary Data 15). Moreover, the other CDK4/6 selective-inhibitor abemaciclib was used to confirm the relationship with $C D K N 2 A / B$ loss. The results showed that IC50s of abemaciclib ( $p=0.0017$, Student's $t$ test) in the CDKN2A or the CDKN2B loss group were significantly lower than in the wild-type group (Supplementary Fig. 5b and Supplementary Data 14), which was similar trend with the results from CDK4/6 inhibitors palbociclib and ribocicilib. On the other hand, mutation profiles of 10 ESCC commercial cell lines are available from Cancer Cell Line Encycloped (CCLE) project (Supplementary Data 16). Among 10 ESCC commercial cell lines, four ones with $C D K N 2 A / 2 B$ wildtype and six ones with $C D K N 2 A / 2 B$ loss were used to validate this biomarker (Fig. 3c). However, the results of drug-sensitivity evaluation showed that there was no significant difference in the IC50s of palbociclib ( $p=0.7981$, Student's $t$ test) and ribociclib ( $p=0.7910$, Student's $t$ test) between commercial cell lines with CDKN2A/2B wild-type and ones with $C D K N 2 A / 2 B$ loss (Fig. $3 d$ ). The data suggest that the potential biomarker of CDK4/6 inhibitors could be validated in ESCC PDCs rather than commercial cell lines.

For further validation of biomarkers of CDK4/6 inhibitors, ZEC127 (CDKN2A/2B loss) and ZEC118 (CDKN2A/2B wildtype) were used to evaluate the sensitivity of CDK4/6 inhibitors using a colony formation assay. The number of ZEC127 (CDKN2A/2B loss) colonies was very significantly reduced at the first $(p<0.001$ and $p<0.001$, Student's $t$ test), for the lowest dose of both palbociclib $(0.25 \mu \mathrm{M})$ and ribociclib $(1 \mu \mathrm{M})$ (Fig. $4 \mathrm{a})$, while the number of ZEC118 (CDKN2A/2B wild-type) colonies maintained at the same level across a series dose, but reduced with higher doses of palbociclib $(5 \mu \mathrm{M})$ and ribociclib $(5 \mu \mathrm{M})$ (Fig. 4b). In addition, the result of ZEC166 (CDKN2A/2B wildtype) was a similar trend to the result of ZEC118 (Supplementary Fig. 5c). These results indicated that ESCC PDCs with CDKN2A or $C D K N 2 B$ loss are sensitive to CDK4/6 inhibitors.

In order to investigate the possible effects of these differences on CDK4/6 inhibitor sensitivity caused by cell cycle checkpoint genes, somatic mutation, and whole transcriptome analyses of ESCC PDCs were performed using targeted deep sequencing and RNAsequencing. We discovered that there are some mutations of cell cycle checkpoint genes, including CDKN2A,CDKN2B, MYC, CCND1, CDK4, RB1, TP53, CHEK1, and CCNE1 in the eight PDCs (Fig. 4c). The messenger RNA (mRNA) expression of these genes in these PDCs is shown in Fig. 4d. Further analyses of the associations of gene somatic mutations and mRNA expression were performed. Interestingly, the expression levels of TP53 nonsynonymous mutations and CHEK1 loss were significantly different between the mutated group and the non-mutated group ( $p=0.0002$ and $p=0.0399$, Wilcoxon's test) (Supplementary Data 17). Most of the cell cycle-related genes (MYC, CCND1, $C D K 4, R B 1$, and CCNE1) were not significantly different in the expression levels (Supplementary Data 17). Moreover, the mRNA expression of $C D K N 2 A$ and $C D K N 2 B$ in the five PDCs harboring $C D K N 2 A$ or $C D K N 2 B$ loss was significantly lower than in the three PDCs with no CNV of CDKN2A and CDKN2B $(p=0.0357$ and $p=0.0357$, Wilcoxon's test, respectively, Supplementary Fig. $5 \mathrm{~d}, \mathrm{e}$ ), indicating that $C D K N 2 A$ or $C D K N 2 B$ loss leads to their depleted expression on the transcriptional level. In addition, the expression of cell cycle checkpoint proteins and their phosphorylation after treating with CDK4/6 inhibitors was detected by means of western blotting. We found that there were reductions of $\mathrm{RB}, \mathrm{pRB}, \mathrm{CDK} 2$, and $\mathrm{pCDK} 2$ in PDCs-ZEC043, 


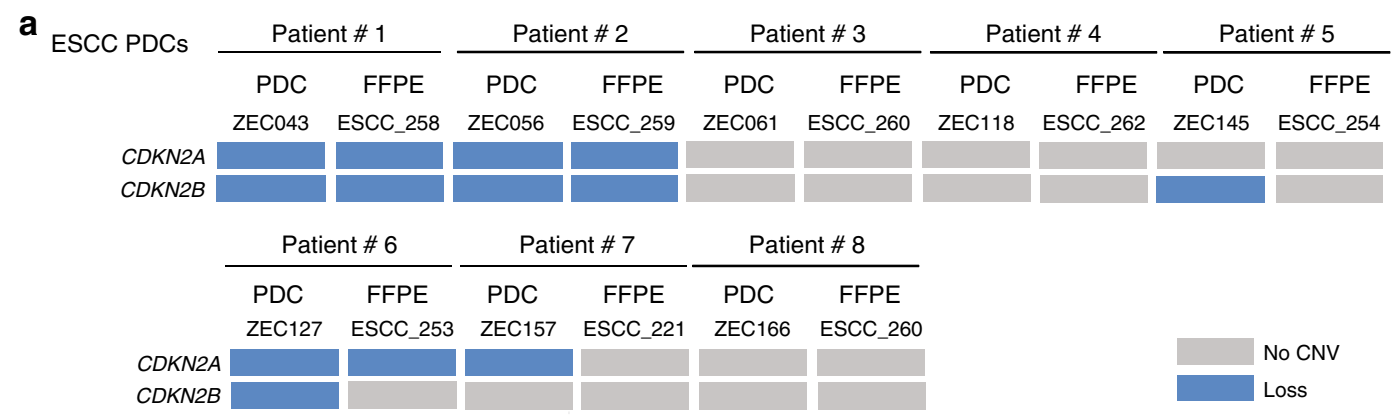

b ESCC PDCs
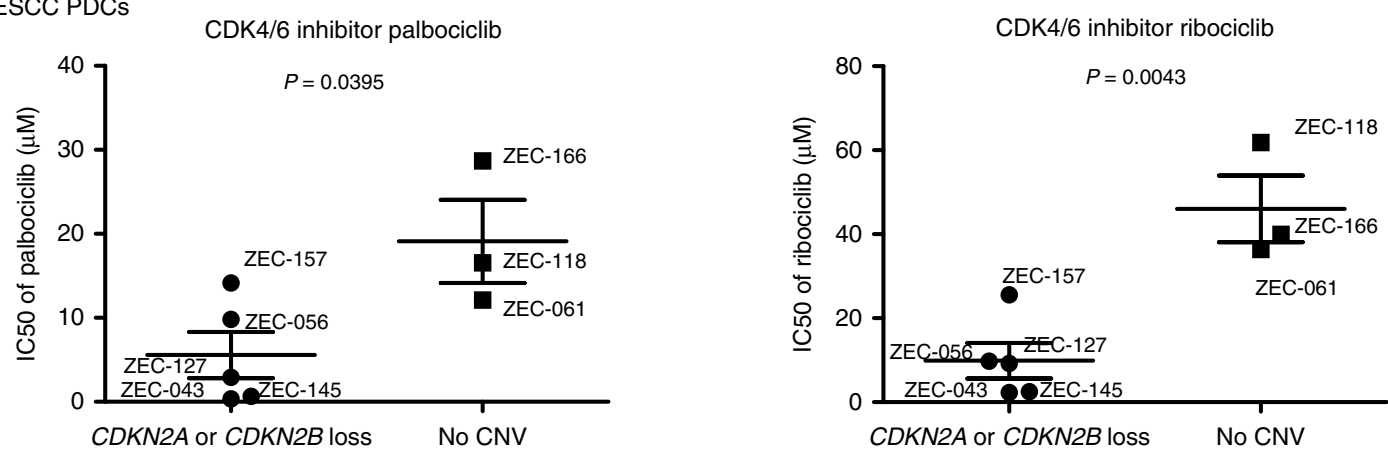

C ESCC commerical cell lines

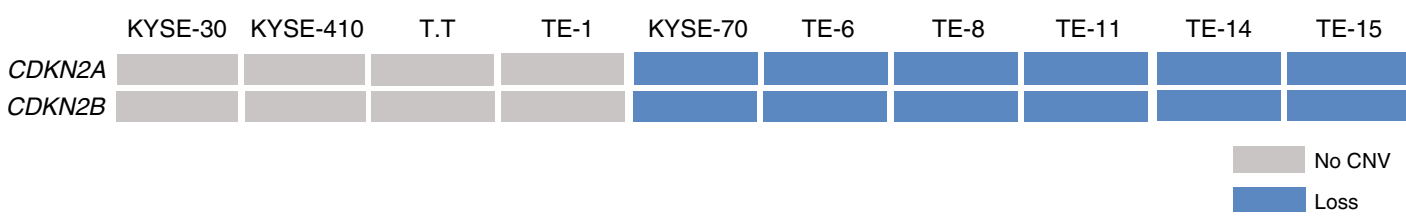

$\mathbf{d}_{\text {ESCC commerical cell lines }}$
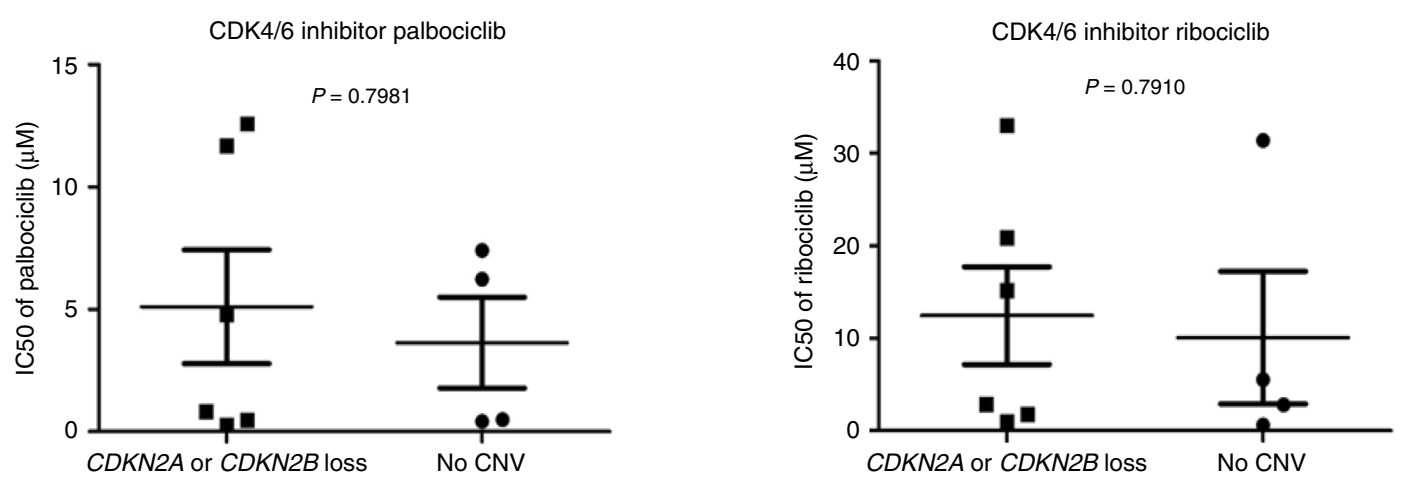

Fig. 3 Validation of the biomarkers in PDCs and commercial cell lines. a The copy number variations (CNVs) of CDKN2A and CDKN2B were shown in eight ESCC PDCs and their corresponding tumor tissue. b Fifty percent growth inhibitory concentrations (IC50s) of palbociclib and ribociclib (LEE011) in eight ESCC PDCs with CDKN2A or CDKN2B loss or with no CNV of CDKN2A and CDKN2B. c The CNVs of CDKN2A and CDKN2B were illustrated in ten commercial cell lines. $\mathbf{d}$ IC50s of palbociclib and ribociclib in ten ESCC commercial cell lines with CDKN2A or CDKN2B loss or with no CNV of CDKN2A and CDKN2B. Error bars correspond to the standard deviation of IC50s. The comparisons between different groups of compound IC50s were performed using Student's $t$ test

ZEC056, and ZEC127 (CDKN2A/2B loss) with increasing dosage of palbociclib (Supplementary Fig. 5f). These data support the idea that biomarkers of CDK4/6 inhibitors for ESCC patients could include a loss of $C D K N 2 A$ or $C D K N 2 B$ rather than mutations of other cell cycle checkpoint genes in vitro.

Validation of the biomarkers of drug sensitivity in vivo. To confirm the results in vivo, ESCC PDC xenograft (PDCX) models were established. The two ESCC PDCs (ZEC145, with $C D K N 2 A / 2 B$ loss, and ZEC166, harboring wild-type $C D K N 2 A / 2 B$ ) were both capable of generating tumor xenografts. Based on previous pre-clinical studies of palbociclib, dosages of 75 and 150 $\mathrm{mg} / \mathrm{kg}$ were chosen for this study ${ }^{29,30}$. As expected, in PDCXZEC145 models with $C D K N 2 A$ and $C D K N 2 B$ loss, palbociclib given at a $75 \mathrm{mg} / \mathrm{kg}$ dose demonstrated a remarkable inhibition of tumor growth (Fig. 5a, b). When the dosage of palbociclib was increased to $150 \mathrm{mg} / \mathrm{kg}$, more obvious regression of the subcutaneous tumors was found (Fig. 5a, b). However, in PDCXZEC166 models with wild-type CDKN2A and CDKN2B, the tumors still progressed in both palbociclib treatment groups 
a
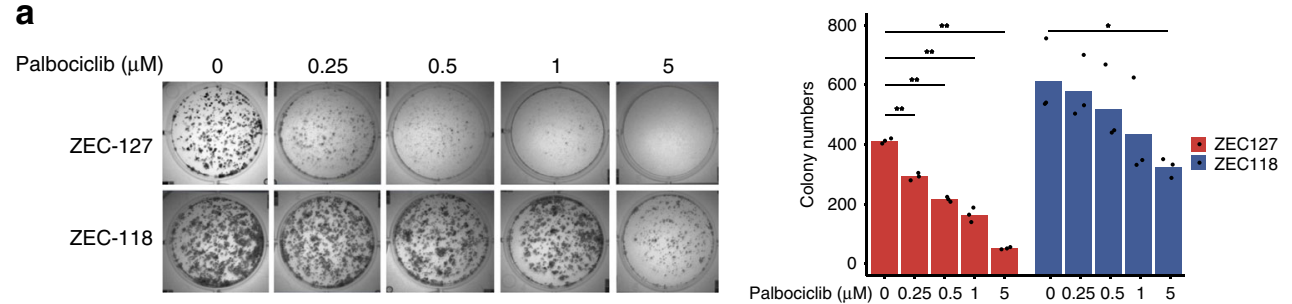

b
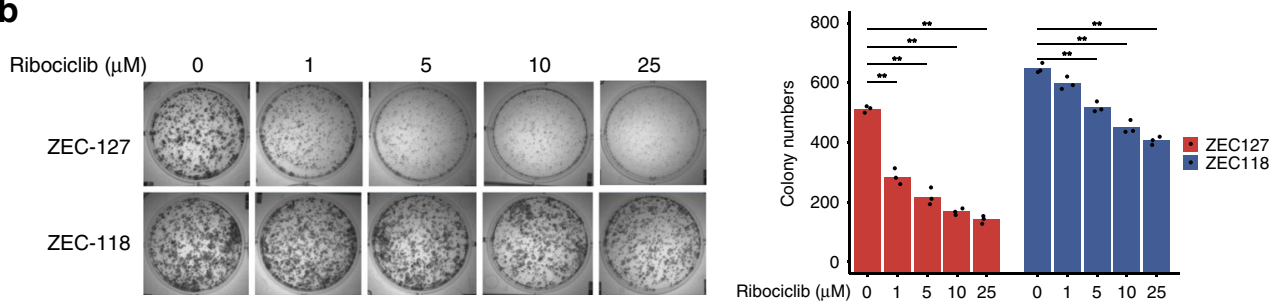

C

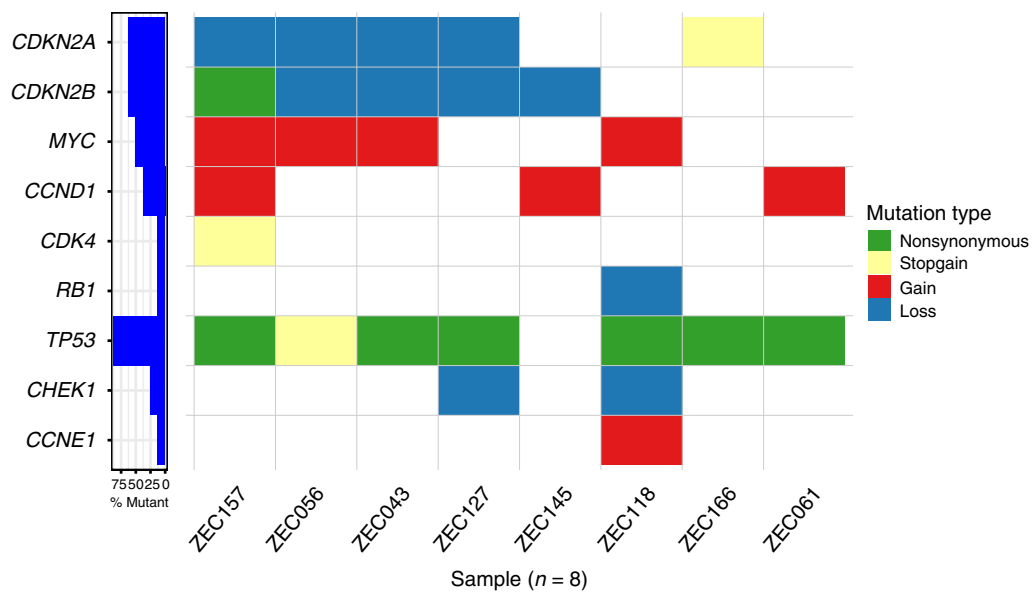

d

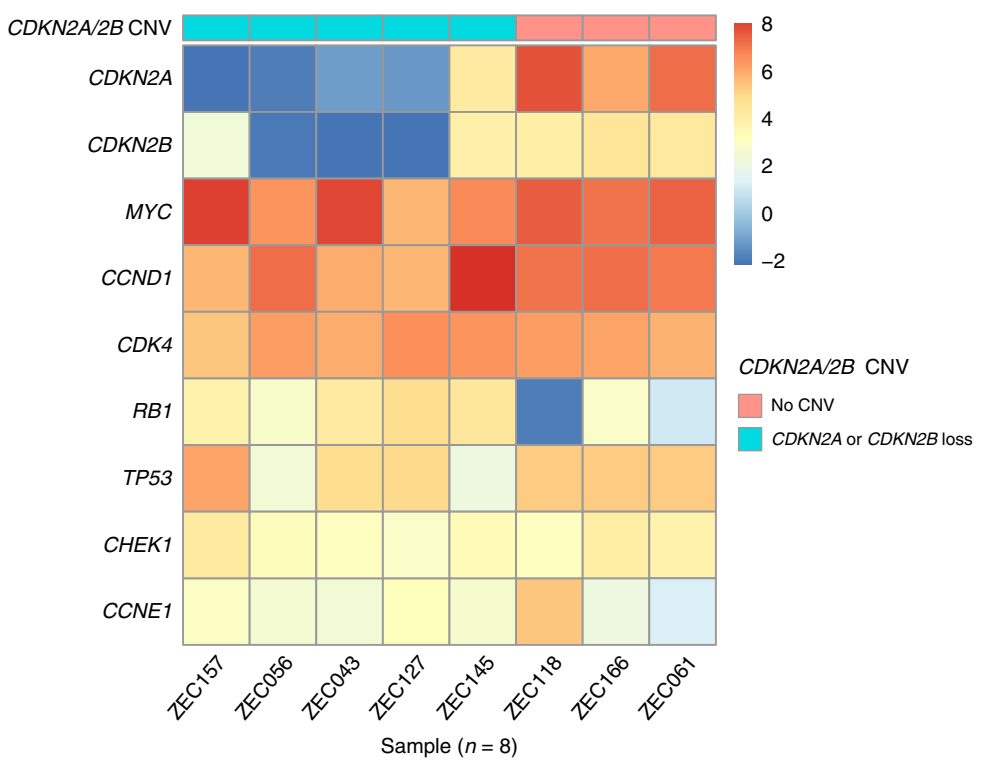

Fig. 4 Validation of the biomarkers of drug sensitivity in vitro. $\mathbf{a}$, b ZEC127 (CDKN2A/2B loss) and ZEC118 (CDKN2A/2B no CNV) were used to evaluate the sensitivity of CDK4/6 inhibitors (palbociclib and ribociclib) using colony formation assays. Three experiments were averaged, and error bars correspond to the standard deviation of colony numbers. The colony number differences between different dose groups of inhibitors were compared using a Student's $t$ test and ${ }^{*} p<0.01$ and ${ }^{* *} p<0.001$. c Mutations of cell cycle checkpoint genes including CDKN2A, CDKN2B, MYC, CCND1, CDK4, RB1, TP53, CHEK1, and CCNE1 were detected in eight ESCC PDCs using targeted deep sequencing. $\mathbf{d}$ Z-score analysis of the expression level of cell cycle checkpoint genes in the eight ESCC PDCs, which carried either CDKN2A or CDKN2B loss, or neither 
a

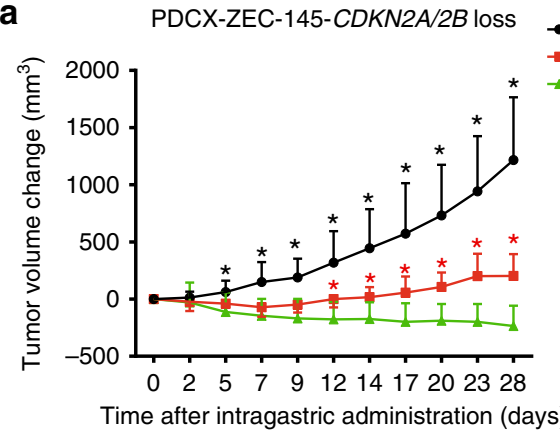

Palbociclib

$0 \mathrm{mg}$ per $\mathrm{kg}$

$(n=10)$

75 mg per kg

$(n=10)$

150 mg per kg
$(n=10)$

C
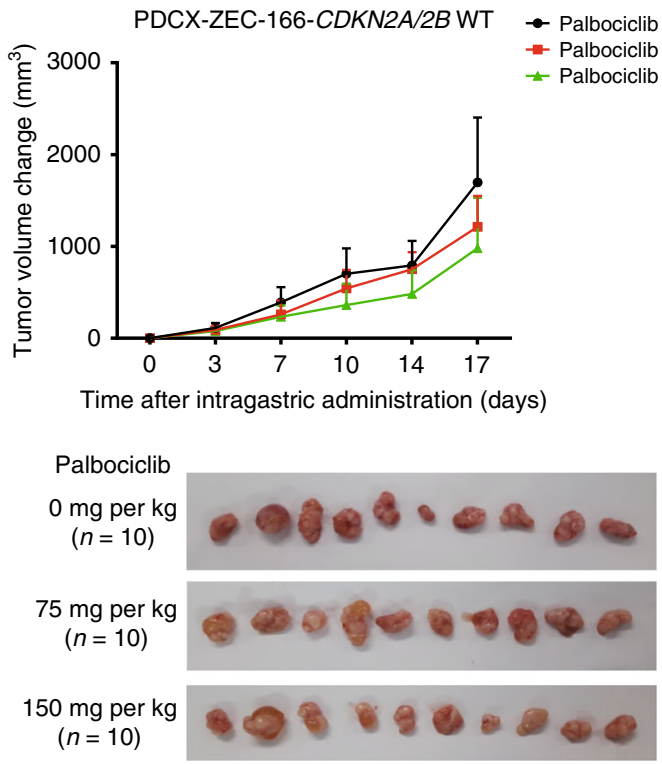

b

- Palbociclib $0 \mathrm{mg}$ per $\mathrm{kg}(n=10)$
- Palbociclib $75 \mathrm{mg}$ per $\mathrm{kg}(n=10)$

- Palbociclib $150 \mathrm{mg}$ per $\mathrm{kg}(n=10)$

PDCX-ZEC-145-CDKN2A/2B loss

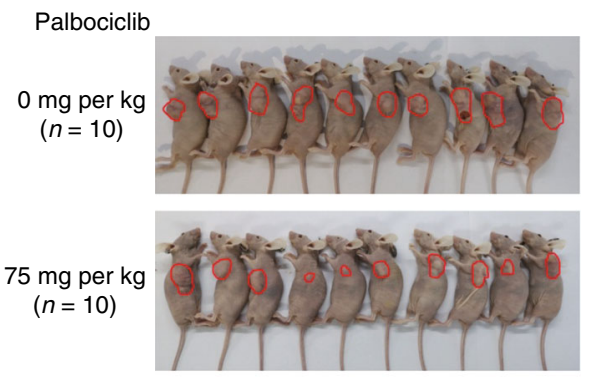

$150 \mathrm{mg}$ per kg $(n=10)$

d

PDCX-ZEC-166-CDKN2A/2B WT

Palbociclib

0 mg per kg

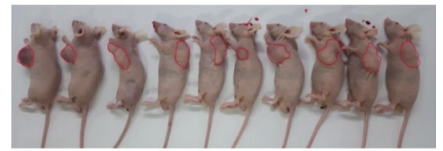

$75 \mathrm{mg}$ per $\mathrm{kg}$
$(n=10)$

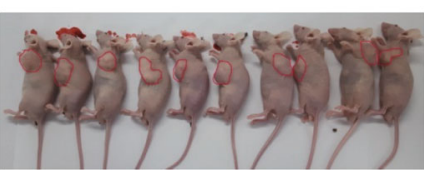

$150 \mathrm{mg}$ per $\mathrm{kg}$ $(n=10)$

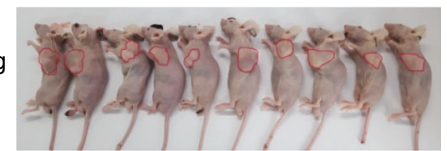

Fig. 5 Validation of the biomarkers of drug sensitivity in vivo. $\mathbf{a}, \mathbf{b}$ Change in tumor volume following treatment with palbociclib at three doses in ZEC145 PDCX CDKN2A/2B loss tumors (28 days) and in ZEC166 PDCX CDKN2A/2B-WT tumors (17 days) (mean \pm s.e.m., $n=10$ ). c, d General picture of ZEC145 PDCXs and ZEC166 PDCXs after intragastric administration of palbociclib (10 mice per dose). Error bars correspond to standard error of the mean of tumor volume. ${ }^{\star} p<0.01$, Student's $t$ test, palbociclib $0 \mathrm{mg} / \mathrm{kg}$ compared with palbociclib $75 \mathrm{mg} / \mathrm{kg}$; ${ }^{\star} p<0.01$, Student's $t$ test, palbociclib $75 \mathrm{mg} / \mathrm{kg}$ compared with palbociclib $150 \mathrm{mg} / \mathrm{kg}$

(Fig. 5c, d). In addition, we observed the most apparent toxicity at the high dose of palbociclib $(150 \mathrm{mg} / \mathrm{kg})$ in both the CDKN2A and $C D K N 2 B$ loss and the wild-type models (Supplementary Fig. 6a, b). In addition, after treatment with the CDK4/6 inhibitor palbocicilib of $75 \mathrm{mg} / \mathrm{kg}$ in vivo, the expression of Foxm1 was downregulated in CDKN2A/2B loss xenografts (PDCX-ZEC145), but up-regulated in $C D K N 2 A / 2 B$ wild-type xenografts (PDCXZEC166), while Smac levels increased in both xenografts. (Supplementary Fig. $6 \mathrm{c}$ ). These data suggest that ESCC PDCX models with $C D K N 2 A$ and $C D K N 2 B$ loss are more sensitive to the CDK4/ 6 inhibitor palbociclib than the PDCX models with wild-type CDKN2A and CDKN2B.

An ESCC PDX model confirms biomarkers of drug sensitivity. A male patient was diagnosed with ESCC in Zhejiang Cancer
Hospital. He had an operation on 23 June 2016. The hematoxylin and eosin (HE) staining of his FFPE tumor tissue was shown in Fig. 6a. He had current smoking status without alcohol intake, and he had no family history of malignant tumors. His tumor was from the distal esophagus. Based on the seventh edition of the American Joint Committee on Cancer (AJCC) staging system for esophageal cancer ${ }^{31}$, he was stage IIIb (Supplementary Data 18). Mutational profiling was carried out by targeted deep sequencing (Supplementary Data 19) and showed that his tumor tissue harbored $C D K N 2 A$ and $C D K N 2 B$ loss. In addition, his tumor tissue was negative for both p15 (coded by CDKN2B) and p16 (coded by CDKN2A) by immunohistochemistry (IHC) staining (Fig. 6b). Unfortunately, he died before he could start taking CDK4/6 inhibitors. However, a PDX model (Z16062301) was successfully created by directly engrafting his surgically resected 
a

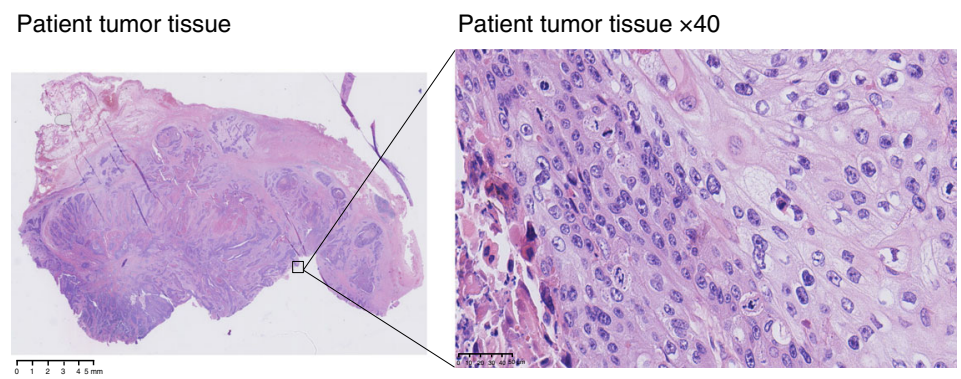

b

p15
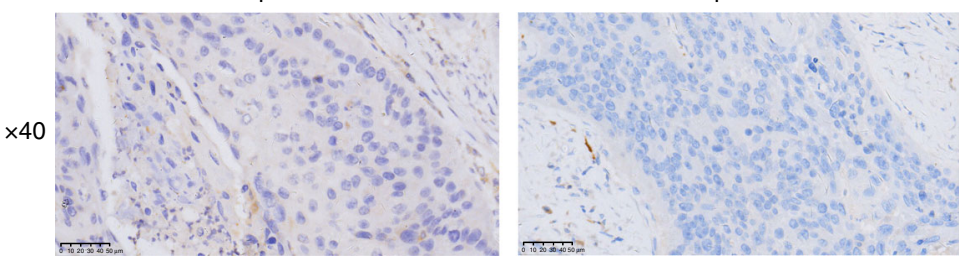

C

ESCC PDX

d (Z16062301)-CDKN2A/2B loss
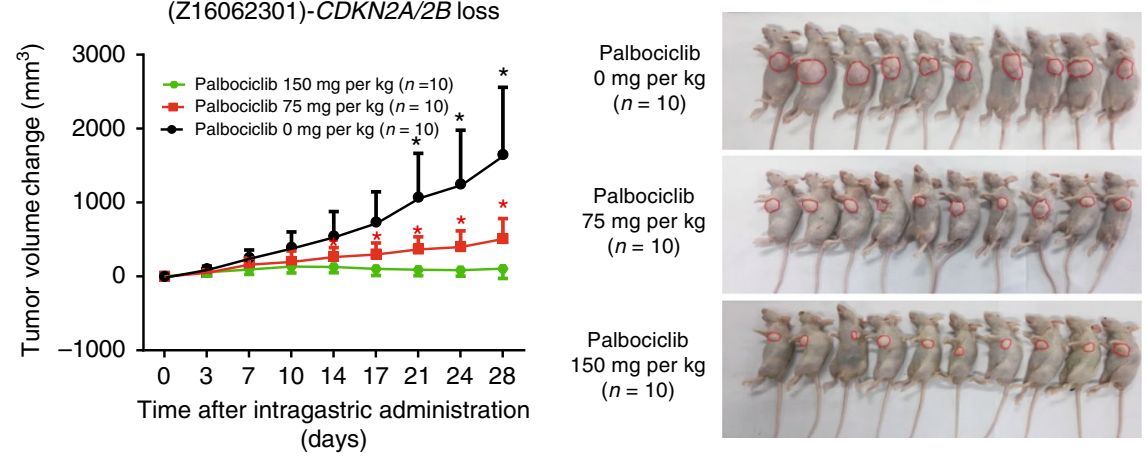

e
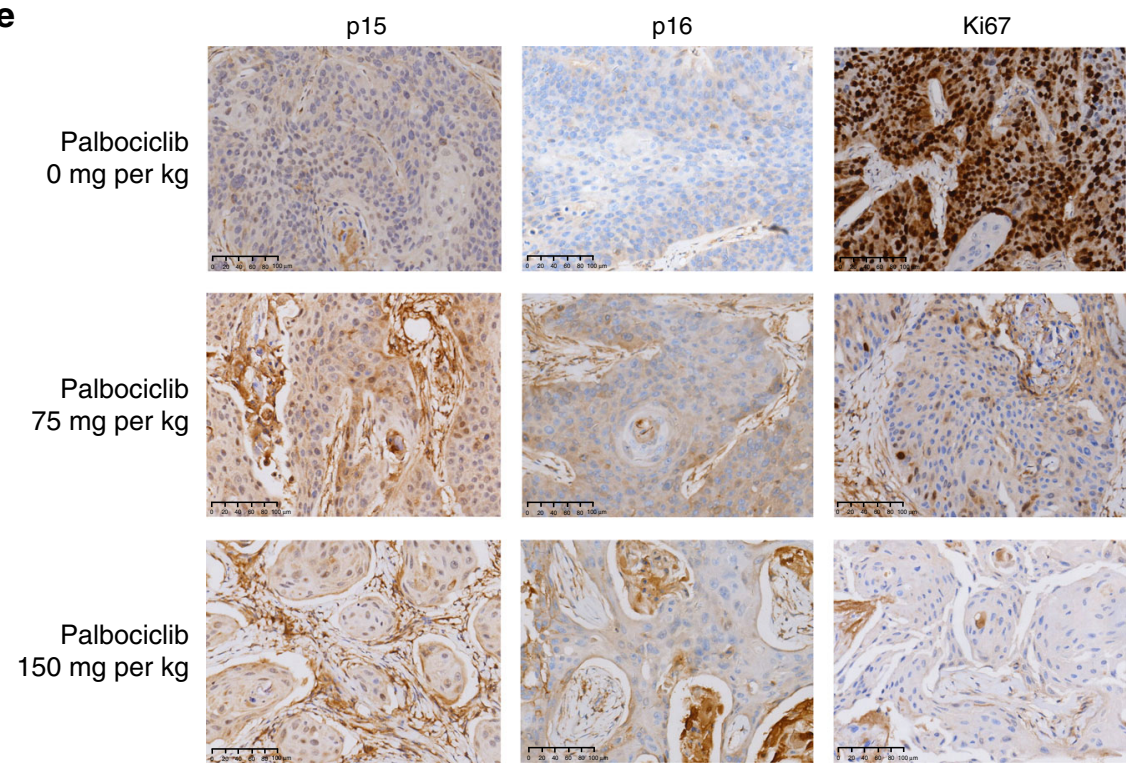

Fig. 6 An ESCC PDX model to confirm the biomarkers of drug sensitivity. a Hematoxylin and eosin staining (HE) results of tumor tissue derived from an ESCC patient are shown. Magnification (right) is $\times 400$. $\mathbf{b}$ The expression levels of p15 and p16 were detected by immunohistochemistry (IHC) staining in the FFPE tumor tissues of this ESCC patient. Magnification (right) is $\times 400$. c Change in tumor volume following treatment with palbociclib in three doses in patient-derived xenografts (PDXs)-Z16062301 (28 days) (mean \pm s.e.m., $n=10$ ). Error bars correspond to standard error of the mean of tumor volume. ${ }^{\star} p<0.01$, Student's $t$ test, palbociclib $0 \mathrm{mg} / \mathrm{kg}$ compared with palbociclib $75 \mathrm{mg} / \mathrm{kg}$; ${ }^{*} p<0.01$, Student's $t$ test, palbociclib $75 \mathrm{mg} / \mathrm{kg}$ compared with palbociclib $150 \mathrm{mg} / \mathrm{kg}$. d General picture of PDXs-Z16062301 after intragastric administration. e IHC staining of FFPE tumor tissue from PDXs treated with different dosages of palbociclib was used to detect the expression of p15, p16, and Ki67. The imaging results of IHC staining with the magnification of $\times 200$ are shown 
tumor tissues into immune-deficient mice. In addition, using targeted deep sequencing, we confirmed that the PDX-Z16062301 model had $C D K N 2 A$ and $C D K N 2 B$ loss.

As expected, in PDX-Z16062301 models, palbociclib treatment at a $75 \mathrm{mg} / \mathrm{kg}$ dose resulted in prominent inhibition of tumor growth with progression (Fig. 6c, d). When the dosage of palbociclib was increased to $150 \mathrm{mg} / \mathrm{kg}$, more notable regression of the subcutaneous tumors was seen (Fig. 6c, d). We also observed the most apparent toxicity at this high dose of palbociclib $(150 \mathrm{mg} / \mathrm{kg})$ in both CDKN2A and CDKN2B loss and wild-type models (Supplementary Fig. 7a). Moreover, IHC staining of FFPE tumor tissue from PDXs treated with different dosages of palbociclib and potential quantification showed that p15 and p16 were almost stain free, and the number of cells that were Ki67 positive was reduced with increasing palbociclib dose (Fig. 6e and Supplementary Data 20). In addition, we found that expression levels of Foxm1 were decreased after treated with CDK4/6 inhibitor palbocicilib of $75 \mathrm{mg} / \mathrm{kg}$ in PDX-Z16062301 (Supplementary Fig. 7b). This mouse study of the ESCC PDX model confirms that $C D K N 2 A$ and $C D K N 2 B$ loss is a biomarker of CDK4/6 inhibitor sensitivity.

\section{Discussion}

To identify the biomarkers of drug sensitivity in a CCLE project and accompanying reports, high-throughput drug screening was employed in commercialized cell lines from multiple types of cancer $^{1-3,32,33}$. The biomarkers of drug sensitivity were systematically identified in that pan-cancer cell model. However, this approach is largely limited because there have been few pancancer biomarkers discovered so far such as NTRK fusion, MSI, and so on ${ }^{34-39}$. A majority of biomarkers are cancer-type-specific. For example, V600E BRAF melanoma but not colorectal cancers were sensitive to $B R A F$-targeting vemurafenib ${ }^{40}$. Amrita Basu et al. ${ }^{3}$ showed that KRAS mutations correlate significantly with sensitivity to navitoclax among colorectal cancer cell lines (CCLs), but not among all CCLs. In our study, we focused on a single cancer type, ESCC, using an approach composed of patientderived models combined with targeted deep sequencing and drug-sensitivity evaluation systems.

We further analyzed the results of identifying the biomarkers of drug sensitivity by overlapping 14 compounds on 27 commercialized ESCC cell lines from the CCLE project and 8 ESCC PDCs from our platform. $P$ values showing gene-drug sensitivity associations were adjusted using FDR correction for multiple testing. The results of the CCLE project showed that there was no gene-drug associations with drug sensitivity established in ESCC commercial cell lines (Supplementary Fig. 8a). Our data from the overlap of 14 compounds revealed an obvious FDR gap between the association of $C D K N 2 A$ or $C D K N 2 B$ loss with palbociclib for drug sensitivity and other gene-drug associations (Supplementary Fig. 8b). This association was further validated in ESCC patientderived models in vitro and in vivo. These discrepant results suggest that commercialized cell line models have limitations in exploring the biomarkers of drug sensitivity, which could be overcome by PDC models. Despite the large cost, the PDC models are valuable and powerful in the identification of potential biomarkers of drug sensitivity.

Apart from association of $C D K N 2 A / 2 B$ loss and CDK4/6 inhibitors, we have adopted this approach to discover other potential drug-genotype associations. We found that two RAC1 gain PDCs were resistant to immunosuppressive agent azathioprine (also named thiopurine and 6-MP), which have been widely used in a variety of clinical conditions for decades, such as Crohn's disease, rheumatic diseases, organ transplantation, and so on ${ }^{41-44}$. Razidlo et al. $^{45}$ found that azathioprine was able to target pancreatic cancer metastasis through inhibition of Rac and Cdc42 signaling. In addition, it was reported that azathioprine induces resistance in hepatoblastoma cells to IGF-1, which leads to autophagy activation ${ }^{46}$. This study was the first to discover that azathioprine-RAC1 gains associations in ESCC, which could help to expand the research of azathioprine in the anti-cancer field. Moreover, we still discovered that KMT2D-mutated PDCs were sensitive to the MLL1 (KMT2A) inhibitor MM-102 in ESCC, which was not found in the CCLE model. MLL1 is one of the six MLL family histone methyltransferases in mammals ${ }^{47,48}$. It was reported that cancer cell proliferation was dramatically lowered by pharmacological inhibition of the MLL1 methyltransferase complex ${ }^{49}$. However, the biomarker of MLL1 inhibitor sensitivity has not been reported. Our discovery of MM-102-KMT2D mutation associations may fill this gap and shed light on MLL1 inhibitor as a promising epigenetic treatment for ESCC patients.

The association of CDKN2A or CDKN2B loss with palbociclib for drug sensitivity was established in this study because we have a balance of five PDCs with $C D K N 2 A$ or $C D K N 2 B$ loss and three PDCs with CDKN2A or CDKN2B wild-type in the drug screening. Other associations were driven by dramatic responses in small numbers of outlier ESCC PDCs. For example, the only ATM-mutated PDC was exquisitely sensitive to the ATM inhibitor KU-55933, and the only DDR1-mutated PDC was sensitive to imatinib targeting $\mathrm{v}$-Abl, c-Kit, and similar targets. Besides that, the only NTRK1-mutated PDC was resistant to MEK1/2 inhibitor trametinib, and the only JAK3-mutated PDC was resistant to JAK1/2 inhibitor Cyt387. These data confirm the need of large panels of ESCC PDCs with a balance of biomarker distribution to capture low-frequency drug-genotype associations. We believe that as PDCs are constantly established, which we are doing now, increasingly more gene-drug associations will be further discovered and validated in ESCC. This powerful approach has paved a way to discover and validate the biomarkers of drug sensitivity in ESCC as well as other cancers.

In this study, we established an approach composed of PDCs combined with targeted deep sequencing and a drug-sensitivity evaluation system in order to explore potential biomarkers of drug sensitivity in ESCC. To further validate these biomarkers of drug sensitivity, patient-derived models, targeted deep sequencing, RNA-sequencing, and drug-sensitivity evaluations were used in vitro and in vivo. Finally, a mouse trial consisting of PDXs was utilized to confirm our results. By this approach, CDKN2A or $C D K N 2 B$ loss was discovered and identified to be a biomarker of CDK4/6 inhibitor sensitivity in ESCC. To our knowledge, this is the first time that researchers have integrated patient-derived models, targeted deep sequencing, RNA-sequencing, drug sensitivity evaluation systems, and mouse studies to identify potential biomarkers of drug sensitivity in relatively large ESCC cohorts. This approach, supported by its effective identification of drug-genotype associations, will lay a foundation for clinical testing of the biomarkers of targeted drug sensitivity.

\section{Methods}

Patient tumor tissue collection. One hundred and sixty-one FFPE tumor tissues and 123 fresh tumor tissues of 284 ESCC patients were obtained from the tissue bank of Zhejiang Cancer Hospital (Table 1 and Supplementary Data 6). Of these tissues, 161 with matched blood samples were used as controls to remove germline variants as a cohort (Table 1). The clinical information and follow-up for 10 years were collected for the 161 patients (Supplementary Data 1). All FFPE samples from this cohort were histologically examined by two senior pathologists independently and were confirmed to contain at least $20 \%$ tumor cells. In this cohort, $87.0 \%(140$ patients) were male, $79.5 \%$ (128 patients) were $\leq 65$ years old, $73.9 \%$ ( 119 patients) had body mass index from 18.5 to $25,77.0 \%$ (124 patients) had current smoking status, $72.7 \%$ (117 patients) had current alcohol intake, $29.8 \%$ (48 patients) had a family history of ESCC, 59.6\% (96 patients) had tumors which were from the middle esophagus, and $73.3 \%$ (118 patients) had tumors that were moderately differentiated. Based on the seventh edition of the AJCC staging system for 
esophageal cancer ${ }^{31}$, one patient $(0.6 \%)$ was stage I, four patients (2.5\%) were stage II, 91 patients $(56.5 \%)$ were stage IIIa, 47 patients $(29.2 \%)$ were stage IIIb, and 18 patients $(11.2 \%)$ were stage IIIc. The median follow-up time for this cohort was 25.6 (range 0-88) months. The 3-year disease-free rate and 3-year survival rate for this cohort were $43.6 \%$ and $43.6 \%$, respectively. The clinical information from an independent cohort of 123 ESCC patients, whose tumor tissues were used for constructing PDCs, is shown in Supplementary Data 6. All samples were collected with written informed consent, and the study was approved by the Institutional Review Board in Zhejiang Cancer Hospital.

Deep sequencing and somatic mutation calling. The targeted panel consists of 365 cancer-related genes and 25 highly re-arranged genes in cancers (Supplementary Data 2). All sequencing assays were performed in 3DMed Medical Laboratory Co., Ltd (Shanghai), which successfully passed the tissue gene mutation testing capabilities Proficiency Testing on NGS solid tumors organized by American Association for pathology (CAP). DNA was isolated from FFPE slides containing at least $20 \%$ tumor cells ${ }^{48}$, PDCs, fresh tumor tissue samples from PDCX models and PDX models, and the blood samples from the corresponding patients. The library was prepared using IDTX gen hybridization buffer for capture and sequenced on an Illumina NextSeq 500.

FastQC software was used to evaluate the quality of sequencing data (http:// www.bioinformatics.bbsrc.ac.uk/projects/fastqc/). Sequence reads from genomic DNA were mapped to human genome (hg19) reference using BWA-MEM ${ }^{50}$, and bam files were further processed by Picard (http://broadinstitute.github.io/picard/) to sort sequences and remove duplicated reads. Somatic SNVs were called by Mutect, and Indels ( $<50 \mathrm{bp}$ ) were identified by Pindel and VarScan ${ }^{49-51}$. Variant calls meeting the following criteria were advanced to further analysis: (1) the minimum coverage in tumors is 30 ; (2) the maximum mutation frequency in normal samples is $\leq 0.03$; (3) the minimum mutation frequency difference between normal and tumor is $\geq 0.05$; (4) the maximum strand bias is $\leq 0.9$; and (5) the minimum support reads of mutation is $\geq 5$. To remove more false positives, our inhouse scripts were developed to filter out spurious SNVs near tandem repeat regions and indel regions. ANNOVAR software was utilized to facilitate variant annotation ${ }^{52}$ and variants with population frequencies $>0.015$ in the 1000 Genomes Project and in all subjects in the NHLBI-ESP Project with 6500 exomes were filtered out. Finally, we only considered variants annotated as nonsynonymous, stop, gain and loss, synonymous, splice site, frameshift deletion and insertion, and non-frameshift deletion and insertion. For the variant calling, the blood samples from the corresponding patients were used as matched normal controls. For somatic CNVs, we applied a series of normalizations to sequencing coverage including matched normal sample, GC content, and segmentation. The coverage in the tumor was normalized to that in the matched normal, further normalization was done by nucleotide composition including GC content, and followed by segmentation and $\log$ ratio estimation, which were similar to ones described in BIC-Seq2 ${ }^{51}$. Segment level CNV was defined as segments with log ratio $>0.7$ or $<-0.7$. The gene level CNV was defined as genes with $>75 \%$ exons overlapping gain/loss segments. R package GenVisR was used to demonstrate somatic mutations spectrum and CNVs across ESCC cohorts ${ }^{52}$.

Establishment and validation of primary cell lines. Tissues collected at the time of surgery and pleural effusions collected from palliative paracentesis were used for primary cell culture. Fresh tumor tissue was rinsed in phosphate-buffered saline (PBS) three times and minced into $0.5-1 \mathrm{~mm}^{3}$ pieces. For pleural effusion, the sediment was harvested and washed in PBS three times. Each sediment was then cultured in Dulbecco's modified Eagle's medium (Gibco) supplemented with 10\% fetal bovine serum (Gibco), $1 \times$ non-essential amino acids (Gibco), $50 \mathrm{U} / \mathrm{mL}$ of penicillin (Gibco) and $50 \mu \mathrm{g} / \mathrm{mL}$ of streptomycin (Gibco), and maintained in a $37^{\circ} \mathrm{C}, 5 \% \mathrm{CO}_{2}$ incubator. After 5 to 7 days, cells were re-plated and designated as passage 0 .

For STR genotyping, genomic DNA was extracted from each primary cell line using a QIAamp DNA Mini Kit (Qiagen Inc., Valencia, CA, USA). Nineteen STR loci (TH01, D12S391, D7S820, CSF1PO, FGA, D5S818, D2S1338, D21S11, D18S51, TPOX, vWA, D8S1179, D3S1358, D13S317, D6S1043, D16S539, Penta E, D19S433, and Penta D) and amelogenin were amplified by PCR and analyzed using an Applied Biosystems 3730xl DNA Analyzer (Applied Biosystems Inc., Foster City, CA, USA). For karyotyping, exponentially growing primary cells were exposed to colchicine $(0.01 \mathrm{mg} / \mathrm{mL})$ for $16 \mathrm{~h}$ and then to hypotonic treatment $(0.075 \mathrm{~mol} / \mathrm{L}$ $\mathrm{KCl})$ for $20 \mathrm{~min}$. After fixation in a methanol and acetic acid mixture (3:1 by volume), cell suspensions were dropped onto ice-cold slides. Slides were then treated in trypsin for 30-60 s and stained with Giemsa. Chromosomes from at least 20 metaphases per sample were analyzed under a microscope.

In vitro cell viability assay with compounds. For cell viability studies, cells were plated in quadruplicate at a density of 4000 cells per well in 96-well plates in normal growth medium and allowed to adhere overnight. The compounds (CDK4/ 6 inhibitors) were then applied to cells at 10 different concentrations in a 3.3-fold dilution series. Cell viability was measured after $72 \mathrm{~h}$ of treatment using the CellTiter-Glo Luminescent Cell Viability Assay (Promega). The concentration of a drug resulting in $50 \%$ inhibition of cell viability (IC50) was calculated from a four- parameter curve analysis. Mean IC50 values and standard deviations of CDK4/6 inhibitors were calculated from four independent experiments for the in vitro validation.

In addition, ten ESCC commercial cell lines used in cell viability assay with compounds were purchased from three different cell banks, including European Collection of Authenticated Cell Cultures (ECACC), Japanese Collection of Research Bioresources (JCRB), and RIKEN BRC cell banks. The vendor of KYSE30 (ECACC, cat. no. 94072011), KYSE-70 (ECACC, cat. no. 94072012), and KYSE410 (ECACC, cat. no. 94072023) is Sigma-Aldrich (Shanghai) Trading Co., Ltd. The vendor of T.T (JCRB, cat. no. JCRB0262), TE-1 (RIKEN, cat. no. RCB1894), TE-6 (RIKEN, cat. no. RCB1950), TE-8 (RIKEN, cat. no. RCB2098), TE-11 (RIKEN, cat. no. RCB2100), TE-14 (RIKEN, cat. no. RCB2101), and TE-15 (RIKEN, cat. no. RCB1951) is 3DHTS Precision Medicine Institute.

Colony formation assay. In total, 2000 cells per plate were seeded onto 6-well plates and treated with palbociclib (Med Chem Express Inc., USA; 0, 0.25, 0.5, 1 , and $5 \mu \mathrm{M}$ ) for about 2 weeks. Then, the cells were washed with PBS twice, fixed with methanol for $10 \mathrm{~min}$, and stained with $0.1 \%$ crystal violet for $15 \mathrm{~min}$. The dishes were then washed with PBS at least three times. Photographs were captured, and cell clones containing $>50$ cells were counted.

Drug sensitivity in vivo. ESCC PDCs $\left(1 \times 10^{7}\right)$ were subcutaneously injected into the backs of male BALB/c nude mice, which were 4 weeks old with a weight of 17-20 g (Shanghai, China; license no., SCXK 2007-0005). Tumor size was monitored every 3 days, and the tumor growth curve and animal weight were recorded accordingly. Tumor volume $(V)$ was calculated by the formula: $V\left(\mathrm{~mm}^{3}\right)=1 / 2$ (length $\times$ width $^{2}$ ). After 7-14 days, when the tumor volume reached $1000 \mathrm{~mm}^{3}$, the mice were killed. Xenograft tumors were then collected and divided into $1-2 \mathrm{~mm}^{3}$ cubes, which were implanted subcutaneously into the left armpit of male BALB/c mice. After 2 weeks, 40 animals harboring $100-150 \mathrm{~mm}^{3}$ tumors were randomized into four groups receiving palbociclib treatment (Group 1, vehicle; Group 2, $75 \mathrm{mg}$ / $\mathrm{kg}$; Group 3, $150 \mathrm{mg} / \mathrm{kg}$; administered by daily gavage). After 28 days or when the tumor volume had reached $1500 \mathrm{~mm}^{3}$, the experiments were ended, and both tumor and blood samples were reserved. Animal experiments were approved by the Institutional Animal Care and Use Committee of Zhejiang Chinese Medicine University (Hangzhou, Zhejiang, China).

Western blot analysis. Cells were cultivated in $60-\mathrm{mm}$ plates for $24 \mathrm{~h}$, prior to treatment with palbociclib (Med Chem Express Inc., USA; 0, 0.5, 2.5, and $12.5 \mu \mathrm{M}$ ) for $48 \mathrm{~h}$. The cells were then harvested and lysed in NP40 buffer with protease inhibitor cocktails. The concentration of total protein was measured using the Bradford colorimetric assay (Bio-Rad Laboratories, USA). Protein expression was detected using $8 \%$ sodium dodecyl sulfate-polyacrylamide gel electrophoresis. Subsequently, $20 \mu \mathrm{g}$ total protein was transferred to polyvinylidene difluoride membranes (Millipore, USA), and the membranes were blocked for $120 \mathrm{~min}$ with freshly prepared $5 \%$ bovine serum albumin in Tris-buffered saline and Tween-20 (TBST). Following this, the membranes were incubated with antibodies at $4{ }^{\circ} \mathrm{C}$ overnight, washed three times with TBST, and incubated with goat anti-rabbit immunoglobulin G-horseradish peroxidase-conjugated antibodies (Google Biology Technology Inc., China). The following antibodies were used: pRb (Ser780) (diluted 1:1000; cat. no. 8180S; Cell Signaling Technology Inc., USA), pRb (Ser807/ 811) (diluted 1:1000; cat. no. 8516S; Cell Signaling Technology Inc., USA), Rb (diluted 1:800; cat. no. 9313S; Cell Signaling Technology Inc., USA), p-CDK2 (diluted 1:1000; cat. no. 2561S; Cell Signaling Technology Inc., USA), CDK2 (diluted 1:1000; cat. no. 2546S; Cell Signaling Technology Inc., USA), cyclin E1 (diluted 1:2000; cat. no. 11554-1-AP; ProteinTech Group Inc., Chicago, IL, USA), CDK4 (diluted 1:2000; cat. no. 11026-1-AP; ProteinTech Group Inc., Chicago, IL USA), CDK6 (diluted 1:2000; cat. no. 14052-1-AP; ProteinTech Group Inc., Chicago, IL, USA), cyclin D (diluted 1:2000; cat. no. 26939-1-AP; ProteinTech Group Inc., Chicago, IL, USA), E2F1 (diluted 1:2000; cat. no. 12171-1-AP; ProteinTech Group Inc., Chicago, IL, USA), Foxm1 (diluted 1:1000; cat. no. 5436S; Cell Signaling Technology Inc., USA), Smac (diluted 1:1000; cat. no. 15108S; Cell Signaling Technology, Inc., USA), tubulin (diluted 1:1000; cat. no. 10068-1-AP; ProteinTech Group Inc., Chicago, IL, USA) and $\beta$-actin (diluted 1:1000; cat. no. 20536-1-AP; ProteinTech Group Inc., Chicago, IL, USA). The uncropped and unprocessed scans of the most important blots were in the Source Data file.

\section{Genomic DNA and total RNA isolation from primary cell lines. ESCC PDCs} were collected from a 100-mm culture dish, followed by lysing in $600 \mu \mathrm{L}$ of Buffer RLT Plus additional $14.3 \mathrm{M} \beta$-mercaptoethanol, and then for simultaneous purification of genomic DNA and total RNA by the AllPrep DNA/RNA Mini Kit according to the manufacturer's instructions (80204, Qiagen, Shanghai, China). The yield and quality of DNA and RNA were analyzed by the Agilent 2100 Bioanalyzer.

Gene expression profiling of primary cell lines. To identify the gene expression profiling of eight ESCC PDCs, RNA-sequencing was performed ${ }^{53}$. Library preparation is the first step. Briefly, after being randomly interrupted, RNAs are converted into a library of cDNA fragments with adaptors attached to both ends of 
each fragment. Subsequently, the molecules in the library, with amplification, are sequenced and short sequences from both ends (paired-end) are obtained. Randomly interrupt mRNA, cDNA fragments synthesis, RNA library construction, hybrid capture, and sequencing was done at WuXi Next CODE (Shanghai, China). Whole transcriptome sequencing was performed using an Illumina HiSeq X Ten. Expression profiling was quantified as fragments per kilobase of transcript per million mapped reads using featureCounts ${ }^{54}$ after alignment using STAR ${ }^{55}$ with RNA-sequencing data.

Establishment of ESCC PDX models. Tumor tissues collected at the time of surgery were used to establish the PDX models. Fresh surgical specimens were immediately rinsed in PBS three times and minced into $\sim 1 \mathrm{~mm}^{3}$ pieces, which were implanted into the male BALB/c nude mice ( $n=5$ per tumor sample; 4 weeks old, 17-20 g weight; Shanghai, China; license no., SCXK 2007-0005). When the tumors reached $\sim 2 \mathrm{~cm}$ at the transplanted position or the mice showed moribund symptoms, nude mice were killed. Xenograft specimens were then collected. These samples with tumorigenic capacity were referred to as "P1." The "P1" samples were serially passaged in vivo to generate the "P2," "P3," and succeeding passages. These were called PDX models. At the same time, the excess tissues were frozen in a cryopreservation liquid containing 49\% Dulbecco's modified Eagle's medium/ nutrient mixture F-12 (Gibco, Shanghai, China), 50\% fetal bovine serum (; Gibco, USA), and 1\% dimethyl sulfoxide (Sigma-Aldrich Co., USA), and then resuscitations of these cells were performed to test their activities.

Statistical analysis. Statistical analyses were performed using SPSS (version 18.0, Chicago, IL). A $\chi^{2}$ test was performed to assess the correlation between the success rate of PDCs and clinicopathological factors. The association between IC50 of CDK4/6 inhibitors and clinical characteristics of ESCC PDC corresponding patients was assessed by multivariate analysis of variance. Data from the experiments were expressed as mean $\pm \mathrm{SD}$, based on a minimum of three independent experiments. The comparisons between different groups of compound IC50, cell colony number, tumor volume, and mice weight were performed using Student's $t$ test, and a $p$ value of $<0.01$ and $<0.001$ was considered significant and very significant. Differences between different groups of gene expression level were compared using Wilcoxon's test, and a $p$ value of $<0.05$ and $<0.01$ was considered significant and very significant. To evaluate the differences of IC50 (the sensitivity to compounds) between mutated and non-mutated groups, two-sided $t$ test was performed with FDR correction for multiple testing. It was to be noted that the test was conducted when the samples in both mutated and non-mutated groups were not $<2$. FDR was set to 1 if one of the groups had only 1 sample. The log-rank test and Kaplan-Meier analyses were performed for DFS and OS. $P<0.05$ was considered statistically significant.

Reporting summary. Further information on research design is available in the Nature Research Reporting Summary linked to this article.

\section{Data availability}

Targeted deep sequencing and RNA-sequencing data have been submitted to NCBI Sequence Read Archive (SRA) (https://www.ncbi.nlm.nih.gov/sra/) with the accession number PRJNA431487. The source data underlying Figs. 3b, 3d, 4a, 4b, 5a, 5c, 6c and Supplementary Fig. 1a, 1b, 5b, 5c, 5 f, 6a, 6b, 6c, 7a, and $7 b$ are provided as a Source Data file. All other data may be found within the main manuscript or supplementary information or available from the authors upon request.

\section{Code availability}

All the related algorithms were described above and additional code used in this study is available upon request.

Received: 27 December 2018; Accepted: 30 September 2019; Published online: 07 November 2019

\section{References}

1. Barretina, J. et al. The Cancer Cell Line Encyclopedia enables predictive modelling of anticancer drug sensitivity. Nature 483, 603-607 (2012).

2. Garnett, M. J. et al. Systematic identification of genomic markers of drug sensitivity in cancer cells. Nature 483, 570-575 (2012).

3. Basu, A. et al. An interactive resource to identify cancer genetic and lineage dependencies targeted by small molecules. Cell 154, 1151-1161 (2013).

4. van Staveren, W. C. et al. Human cancer cell lines: experimental models for cancer cells in situ? For cancer stem cells? Biochim. Biophys. Acta 1795, 92-103 (2009).

5. Daniel, V. C. et al. A primary xenograft model of small-cell lung cancer reveals irreversible changes in gene expression imposed by culture in vitro. Cancer Res. 69, 3364-3373 (2009).
6. Byrne, A. T. et al. Interrogating open issues in cancer precision medicine with patient-derived xenografts. Nat. Rev. Cancer 17, 254-268 (2017).

7. Pandrangi, S. L. et al. Establishment and characterization of two primary breast cancer cell lines from young Indian breast cancer patients: mutation analysis. Cancer Cell Int. 14, 14 (2014).

8. Zhang, J. et al. Establishment and characterization of esophageal squamous cell carcinoma patient-derived xenograft mouse models for preclinical drug discovery. Lab. Invest. 94, 917-926 (2014).

9. Zhang, X. C. et al. Establishment of patient-derived non-small cell lung cancer xenograft models with genetic aberrations within EGFR, KRAS and FGFR1: useful tools for preclinical studies of targeted therapies. J. Transl. Med. 11, 168 (2013).

10. Kim, M. J. et al. Establishment and characterization of 6 novel patient-derived primary pancreatic ductal adenocarcinoma cell lines from Korean pancreatic cancer patients. Cancer Cell Int. 17, 47 (2017).

11. Dangles-Marie, V. et al. Establishment of human colon cancer cell lines from fresh tumors versus xenografts: comparison of success rate and cell line features. Cancer Res. 67, 398-407 (2007).

12. Gao, Q. et al. Cell culture system for analysis of genetic heterogeneity within hepatocellular carcinomas and response to pharmacologic agents. Gastroenterology 152, 232-242.e4 (2017).

13. Dobrolecki, L. E. et al. Patient-derived xenograft (PDX) models in basic and translational breast cancer research. Cancer Metastasis Rev. 35, 547-573 (2016).

14. Suarez, C. D. \& Littlepage, L. E. Patient-derived tumor xenograft models of breast cancer. Methods Mol. Biol. 1406, 211-223 (2016).

15. Krepler, C. et al. Personalized preclinical trials in BRAF inhibitor-resistant patient-derived xenograft models identify second-line combination therapies. Clin. Cancer Res. 22, 1592-1602 (2016).

16. Chen, W. et al. Cancer statistics in China, 2015. CA Cancer J. Clin. 66, 115-132 (2016).

17. Pennathur, A. et al. Oesophageal carcinoma. Lancet 381, 400-412 (2013).

18. Gao, Y. B. et al. Genetic landscape of esophageal squamous cell carcinoma. Nat. Genet. 46, 1097-1102 (2014).

19. Lin, D. C. et al. Genomic and molecular characterization of esophageal squamous cell carcinoma. Nat. Genet. 46, 467-473 (2014).

20. Song, Y. et al. Identification of genomic alterations in oesophageal squamous cell cancer. Nature 509, 91-95 (2014).

21. Zhang, L. et al. Genomic analyses reveal mutational signatures and frequently altered genes in esophageal squamous cell carcinoma. Am. J. Hum. Genet. 96, 597-611 (2015).

22. Cheng, C. et al. Whole-genome sequencing reveals diverse models of structural variations in esophageal squamous cell carcinoma. Am. J. Hum. Genet. 98, 256-274 (2016).

23. Qin, H. D. et al. Genomic characterization of esophageal squamous cell carcinoma reveals critical genes underlying tumorigenesis and poor prognosis. Am. J. Hum. Genet. 98, 709-727 (2016).

24. Cancer Genome Atlas Research et al. Integrated genomic characterization of oesophageal carcinoma. Nature 541, 169-175 (2017).

25. Harttrampf, A. C. et al. Molecular Screening for Cancer Treatment Optimization (MOSCATO-01) in pediatric patients: a single-institutional prospective molecular stratification trial. Clin. Cancer Res. 23, 6101-6112 (2017).

26. Baik, C. S., Myall, N. J. \& Wakelee, H. A. Targeting BRAF-mutant non-small cell lung cancer: from molecular profiling to rationally designed therapy. Oncologist 22, 786-796 (2017).

27. Chandrani, P. et al. Drug-sensitive FGFR3 mutations in lung adenocarcinoma. Ann. Oncol. 28, 597-603 (2017).

28. Feng, F. et al. Guidance to rational use of pharmaceuticals in gallbladder sarcomatoid carcinoma using patient-derived cancer cells and whole exome sequencing. Oncotarget 8, 5349-5360 (2017).

29. Fry, D. W. et al. Specific inhibition of cyclin-dependent kinase $4 / 6$ by PD 0332991 and associated antitumor activity in human tumor xenografts. Mol. Cancer Ther. 3, 1427-1438 (2004)

30. Baughn, L. B. et al. A novel orally active small molecule potently induces G1 arrest in primary myeloma cells and prevents tumor growth by specific inhibition of cyclin-dependent kinase 4/6. Cancer Res. 66, 7661-7667 (2006).

31. Edge, B. E. et al. AJCC Cancer Staging Manual 7th edn (Springer, New York, 2010).

32. Vanden Heuvel, J. P., Bullenkamp, J., Reproducibility Project: Cancer B. Registered report: systematic identification of genomic markers of drug sensitivity in cancer cells. Elife 5, e13620 (2016).

33. Vanden Heuvel, J. P. et al. Replication study: systematic identification of genomic markers of drug sensitivity in cancer cells. Elife 7, e29747 (2018).

34. Cocco, E., Scaltriti, M. \& Drilon, A. NTRK fusion-positive cancers and TRK inhibitor therapy. Nat. Rev. Clin. Oncol. 15, 731-747 (2018).

35. Liu, D. et al. Entrectinib: an orally available, selective tyrosine kinase inhibitor for the treatment of NTRK, ROS1, and $A L K$ fusion-positive solid tumors. Ther. Clin. Risk Manag. 14, 1247-1252 (2018). 
36. Kheder, E. S. \& Hong, D. S. Emerging targeted therapy for tumors with NTRK fusion proteins. Clin. Cancer Res. 24, 5807-5814 (2018).

37. Le, D. T. et al. PD-1 blockade in tumors with mismatch-repair deficiency. $N$. Engl. J. Med. 372, 2509-2520 (2015).

38. Le, D. T. et al. Mismatch repair deficiency predicts response of solid tumors to PD-1 blockade. Science 357, 409-413 (2017).

39. Asaoka, Y., Ijichi, H. \& Koike, K. PD-1 blockade in tumors with mismatchrepair deficiency. N. Engl. J. Med. 373, 1979 (2015).

40. Prahallad, A. et al. Unresponsiveness of colon cancer to BRAF(V600E) inhibition through feedback activation of EGFR. Nature 483, 100-103 (2012).

41. Tiede, I. et al. CD28-dependent Rac1 activation is the molecular target of azathioprine in primary human CD4+ T lymphocytes. J. Clin. Invest. 111, $1133-1145$ (2003).

42. Marinkovic, G. et al. Inhibition of GTPase Rac1 in endothelium by 6mercaptopurine results in immunosuppression in nonimmune cells: new target for an old drug. J. Immunol. 192, 4370-4378 (2014).

43. Axelrad, J. E., Lichtiger, S. \& Yajnik, V. Inflammatory bowel disease and cancer: The role of inflammation, immunosuppression, and cancer treatment. World J. Gastroenterol. 22, 4794-4801 (2016).

44. Zochowska, D. et al. Determination of concentrations of azathioprine metabolites 6-thioguanine and 6-methylmercaptopurine in whole blood with the use of liquid chromatography combined with mass spectrometry. Transpl. Proc. 48, 1836-1839 (2016).

45. Razidlo, G. L. et al. Targeting pancreatic cancer metastasis by inhibition of Vav1, a driver of tumor cell invasion. Cancer Res. 75, 2907-2915 (2015).

46. Hernandez-Breijo, B. et al. Azathioprine desensitizes liver cancer cells to insulin-like growth factor 1 and causes apoptosis when it is combined with bafilomycin A1. Toxicol. Appl. Pharm. 272, 568-578 (2013).

47. Cao, F. et al. Targeting MLL1 H3K4 methyltransferase activity in mixedlineage leukemia. Mol. Cell 53, 247-261 (2014).

48. Chen, Y. et al. MLL2, not MLL1, plays a major role in sustaining MLLrearranged acute myeloid leukemia. Cancer Cell 31, 755-770 e6 (2017).

49. Zhu, J. et al. Gain-of-function p53 mutants co-opt chromatin pathways to drive cancer growth. Nature 525, 206-211 (2015).

50. Li, H. \& Durbin, R. Fast and accurate short read alignment with Burrows-Wheeler transform. Bioinformatics 25, 1754-1760 (2009).

51. Casey, B. J. et al. Behavioral and neural correlates of delay of gratification 40 years later. Proc. Natl Acad. Sci. USA 108, 14998-15003 (2011)

52. Skidmore, Z. L. et al. GenVisR: Genomic Visualizations in R. Bioinformatics 32, 3012-3014 (2016).

53. Kohli, M. et al. Androgen receptor variant AR-V9 is coexpressed with AR-V7 in prostate cancer metastases and predicts abiraterone resistance. Clin. Cancer Res. 23, 4704-4715 (2017).

54. Liao, Y., Smyth, G. K. \& Shi, W. featureCounts: an efficient general purpose program for assigning sequence reads to genomic features. Bioinformatics $\mathbf{3 0}$, 923-930 (2014).

55. Dobin, A. et al. STAR: ultrafast universal RNA-seq aligner. Bioinformatics 29, $15-21(2013)$

\section{Acknowledgements}

This study was partially supported by the National Health Commission Scientific Research Fund-Zhejiang Medical and Health Major Science and Technology Program (WKJ-ZJ-1902 to D.S.), the National Natural Science Foundation of China (Grant No 81472203 and 81972917 to D.S.) and the High-Level Creative and Innovative Health
Talent Program of Zhejiang Province (to D.S.). We thank all the patients and their families for their support of this study. We also thank Nishihira Tetsuro for his authorization of our use of ESCC cell lines, including TE-1, TE-6, TE-8, TE-11, TE-14 and TE-15. We thank Hua Dong, Changgen Ding, Yinming Jiao, Zisong Zhou, Xiaoya $\mathrm{Xu}$, and Zishuo Chen for their assistance in conducting the experiments in this study.

\section{Author contributions}

D.S., D.Z., and J.J. contributed equally. D.S. and W.M. conceived the study, designed the experiments, analyzed the data, and wrote the manuscript. A.F. edited the manuscript. D.Z., J.J., J. Wu, K.C., Z.X, L.Y., Y.L., F.Z., G.C., J.-Y.L., M. Huang, J. Wang, K.W., J.Z., F. L., and L.X. supervised data analyses and performed the experiments. D.Z., M. Han, B.L., and J.J. performed bioinformatics and statistics analyses. D.S., W.M., K.C., F.Z., J.J., G.C., and Y. L. provided clinical samples, and coordinated and performed pathology review. All authors had access to the study data and reviewed and approved the final manuscript.

\section{Competing interests}

The authors declare the following competing interests: L.X., F.L., J.-Y.L., Y.L., and Z.X. report personal fees from 3D Medicines Inc. and have ownership interest (including patents) in the same, outside the submitted work. M. Han, B.L., D.Z., and J. Wang report personal fees from 3D Medicines Inc., outside the submitted work. Z.J. reports personal fees from BMS, AstraZeneca, Geneplus, OrigMed, and Innovent, as well as a 678 grant from Merck, outside the submitted work. No competing interests were disclosed by the other authors.

\section{Additional information}

Supplementary information is available for this paper at https://doi.org/10.1038/s41467 019-12846-7.

Correspondence and requests for materials should be addressed to D.S. or W.M.

Peer review information Nature Communications thanks Jesus M. Paramio and the other, anonymous, reviewer(s) for their contribution to the peer review of this work.

Reprints and permission information is available at http://www.nature.com/reprints

Publisher's note Springer Nature remains neutral with regard to jurisdictional claims in published maps and institutional affiliations.

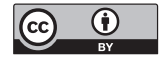

Open Access This article is licensed under a Creative Commons Attribution 4.0 International License, which permits use, sharing, adaptation, distribution and reproduction in any medium or format, as long as you give appropriate credit to the original author(s) and the source, provide a link to the Creative Commons license, and indicate if changes were made. The images or other third party material in this article are included in the article's Creative Commons license, unless indicated otherwise in a credit line to the material. If material is not included in the article's Creative Commons license and your intended use is not permitted by statutory regulation or exceeds the permitted use, you will need to obtain permission directly from the copyright holder. To view a copy of this license, visit http://creativecommons.org/ licenses/by/4.0/.

(c) The Author(s) 2019 